\title{
ON THE SECOND COEFFICIENT REGION FOR BOUNDED UNIVALENT FUNCTIONS
}

\author{
RONALD KORTRAM and OLLI TAMMI
}

\section{Introduction}

Consider the class $S(b)$ of bounded univalent functions $f$ defined in the unit disc $U=\{z \in C|| z \mid<1\}$ and normalized as follows:

$$
\left\{\begin{array}{l}
f(z)=b z+b_{2} z^{2}+\ldots, \\
a_{n}=\frac{b_{n}}{b} \quad\left(n=1,2, \ldots ; b_{1}=b\right), \\
|f(z)|<1, \\
b \text { is constant } \in(0,1] .
\end{array}\right.
$$

For these functions the Power inequality, or $P_{N}$-inequality, holds in its quadratic form

$$
\sum_{-N}^{\infty} k\left|y_{k}\right|^{2}+2 \operatorname{Re}\left(x_{0} y_{0}\right) \leqq \sum_{-N}^{N} k\left|x_{k}\right|^{2},
$$

derived in [2] under the unessential restriction $x_{0} \in R$. The numbers $x_{v}$ are supposed to be free complex parameters, determining the combinations

$$
y_{k}=\sum_{v=-N}^{k} x_{v} c_{p k} \quad(k \geqq-N) .
$$

Here the Power-coefficients $c_{r k}$ are determined by the defining conditions

$$
\left\{\begin{array}{l}
f(z)^{p}=\sum_{k=\nu}^{\infty} c_{v k} z^{k} \quad(v= \pm 1, \pm 2, \ldots), \\
\log \frac{f(z)}{z}=\sum_{k=0}^{\infty} c_{0 k} z^{k},
\end{array}\right.
$$

and the numbers $y_{v}$ are obtained by the aid of the generating function 


$$
g(w)=x_{0} \log w+\sum_{-N}^{N} x_{\nu} w^{v} \quad\left(v \neq 0 \text { in } \sum^{\prime}\right),
$$

as coefficients of the development

$$
g(f(z))=x_{0} \log z+\sum_{k=-N}^{\infty} y_{k} z^{k} .
$$

In [2] there is the bilinear form of the $P_{N}$-inequality

$$
-\operatorname{Re}\left\{\sum_{1}^{N} k\left(y_{-k} y_{k}+x_{-k} x_{k}\right)\right\} \leqq \sum_{1}^{N} k\left(\left|y_{-k}\right|^{2}+\left|x_{k}\right|^{2}\right)
$$

true on the condition that $\operatorname{Re}\left(\bar{x}_{0} y_{0}\right)=0$. We used the bilinear $P_{3}$. inequality in [3] for the maximizing of $\left|a_{4}\right|$ in $S(b)$ by choosing the coefficients $x_{v}$ in a special optimal way. The same optimization worked also when the first coefficient region $\left(a_{3}, a_{2}\right)$ was studied by the aid of the quadratic $P_{1}$-inequality [1]. Similarly, the optimized bilinear $P_{3}$-inequality was able to give some information of the region $\left(a_{4}, a_{3}, a_{2}\right)$ on the condition that all the coefficients $a_{2}, a_{3}, a_{4}$ were supposed to be real.

In the present paper we will make use of the optimization principle in the bilinear $P_{3}$ inequality when applied to the function $F \in S\left(b^{1 / 2}\right)$, where $F(z)=f\left(z^{2}\right)^{1 / 2}, f \in S(b)$. Our aim is to prove that the result concerning $\left(a_{3}, a_{2}\right)$ can be generalized: The given pair $\left(a_{3}, a_{2}\right)$ determines a disc as a range of $a_{4}$.

\section{The optimized bilinear $P_{3}$-inequality for $f\left(z^{2}\right)^{1 / 2}$}

Form first the $P_{3}$-inequality for the function $F(z)=f\left(z^{2}\right)^{1 / 2}$. According to [3], introduce the parameters $u_{v}$,

$$
u_{v}=-v y_{-v} \quad(v=1,2,3)
$$

and apply the symmetric choice

$$
x_{-\nu}=-\bar{x}_{\nu} \quad(v=1,2,3)
$$

together with

$$
x_{0}=x_{2}=0 \text {, }
$$

which agrees with the odd character of $F$. Because this implies

$$
u_{2}=0 ; \quad y_{0}=y_{2}=0,
$$

we obtain for the $P_{3}$-inequality the quadratic truncated form

$$
\left|y_{1}\right|^{2}+3\left|y_{3}\right|^{2} \leqq\left|u_{1}\right|^{2}+\frac{1}{3}\left|u_{3}\right|^{2}
$$


with

$$
x_{3}=\frac{1}{3} b^{3 / 2} \bar{u}_{3}, \quad x_{1}=b^{1 / 2} \overline{\left(u_{1}+\frac{1}{2} a_{2} u_{3}\right)} .
$$

A comparison to (1) shows that equality here requires $y_{4}=y_{5}=\ldots=0$, i.e. for the extremal $F$,

$$
-\frac{1}{3} b^{3 / 2} u_{3} F^{-3}+\frac{1}{3} b^{3 / 2} \bar{u}_{3} F^{3}-b^{1 / 2}\left(u_{1}+\frac{1}{2} a_{2} u_{3}\right) F^{-1}-b^{1 / 2} \overline{\left(u_{1}+\frac{1}{2} a_{2} u_{3}\right)} F
$$

$$
=-\frac{1}{3} u_{3} z^{-3}+y_{3} z^{3}-u_{1} z^{-1}+y_{1} z
$$

holds necessarily. Here

$$
\left\{\begin{array}{l}
y_{1}=a_{1} u_{1}+b \bar{u}_{1}+d_{1} u_{3}+e_{1} \bar{u}_{3}, \\
y_{3}=d_{1} u_{1}+\bar{e}_{1} \bar{u}_{1}+d_{3} u_{3}+e_{3} \bar{u}_{3},
\end{array}\right.
$$

where

$$
\left\{\begin{array}{l}
a_{1}=\frac{1}{2} a_{2} \\
d_{1}=\frac{1}{2} a_{3}-\frac{3}{4} a_{2}^{2}, \\
e_{1}=\frac{1}{2} b \bar{a}_{2}, \\
e_{3}=\frac{1}{4} b\left|a_{2}\right|^{2}+\frac{1}{3} b^{3}, \\
d_{3}=\frac{1}{2} a_{4}-a_{2} a_{3}+\frac{13}{2} a_{2}^{3} .
\end{array}\right.
$$

Because the bilinear form (2) is obtained from the quadratic form (1) by the aid of Schwarz's inequality, we see (cf. [2], (57)) that equality is preserved if, together with the symmetric choice $x_{-v}=-\bar{x}_{\nu}$, we have

$$
\left\{\begin{array}{l}
y_{1}=-\bar{y}_{-1} \\
y_{3}=-\bar{y}_{-3} .
\end{array}\right.
$$

The experience gained in [1] in connection with the quadratic $P_{1}$-inequality justifies our expecting (6) to hold in the optimized case for $P_{3}$, too. Thus, we will base our studies on the bilinear $P_{3}$-inequality

$$
\operatorname{Re}\left(u_{1} y_{1}+u_{3} y_{3}\right) \leqq\left|u_{1}\right|^{2}+\frac{1}{3}\left|u_{3}\right|^{2}
$$

i.e.

$$
\begin{aligned}
G & =\frac{1}{2} a_{1} u_{1}^{2}+\frac{1}{2} \bar{a}_{1} \bar{u}_{1}^{2}+(b-1)\left|u_{1}\right|^{2}+d_{1} u_{1} u_{3}+\bar{d}_{1} \bar{u}_{1} u_{3} \\
& =e_{1} u_{1} \bar{u}_{3}+\bar{e}_{1} \bar{u}_{1} u_{3}+\operatorname{Re}\left(d_{3} u_{3}^{2}\right)+\left(e_{3}-\frac{1}{3}\right)\left|u_{3}\right|^{2} \leqq 0
\end{aligned}
$$

Now, keep first $u_{3}$ constant and optimize with respect to

$$
u_{1}=x+i y
$$

by determining $u_{1}$ from $\partial G / \partial x=\partial G / \partial y=0$. We will make use of the formulae 


$$
\begin{cases}\frac{\partial\left|u_{1}\right|^{2}}{\partial x}=u_{1}+\bar{u}_{1}, & \frac{\partial\left|u_{1}\right|^{2}}{\partial y}=i\left(\bar{u}_{1}-u_{1}\right), \\ \frac{\partial u_{1}}{\partial x}=\frac{\partial \bar{u}_{1}}{\partial x}=1 ; & \frac{\partial u_{1}}{\partial y}=-\frac{\partial \bar{u}_{1}}{\partial y}=i,\end{cases}
$$

to give

$$
\left\{\begin{aligned}
\frac{\partial G}{\partial x}= & a_{1} u_{1}+\bar{a}_{1} \bar{u}_{1}+(b-1)\left(u_{1}+\bar{u}_{1}\right)+d_{1} u_{3}+\bar{d}_{1} \bar{u}_{3} \\
& +e_{1} \bar{u}_{3}+\bar{e}_{1} u_{3}=0, \\
\frac{1}{i} \frac{\partial G}{\partial i}= & a_{1} u_{1}-\bar{a}_{1} \bar{u}_{1}+(b-1)\left(\bar{u}_{1}-u_{1}\right)+d_{1} u_{3}-\bar{d}_{1} \bar{u}_{3} \\
& +e_{1} \bar{u}_{3}-\bar{e}_{1} u_{3}=0 .
\end{aligned}\right.
$$

This is equivalent to

$$
a_{1} u_{1}-(1-b) \bar{u}_{1}+d_{1} u_{3}+e_{1} \bar{u}_{3}=0 .
$$

In the equality case

$$
\begin{aligned}
0= & \frac{1}{2}\left(x \frac{\partial G}{\partial x}+y \frac{\partial G}{\partial y}\right)=\frac{1}{2} a_{1} u_{1}^{2}+\frac{1}{2} \bar{a}_{1} \bar{u}_{1}^{2}+(b-1)\left|u_{1}\right|^{2} \\
& +\frac{1}{2}\left(d_{1} u_{3} u_{1}+\bar{d}_{1} \bar{u}_{3} \bar{u}_{1}+e_{1} \bar{u}_{3} u_{1}+\bar{e}_{1} u_{3} \bar{u}_{1}\right)
\end{aligned}
$$

i.e.

$$
\begin{aligned}
G= & \frac{1}{2}\left(d_{1} u_{1} u_{3}+\bar{d}_{1} \bar{u}_{1} \bar{u}_{3}+e_{1} u_{1} \bar{u}_{3}+\bar{e}_{1} \bar{u}_{1} u_{3}\right) \\
& +\operatorname{Re}\left(d_{3} u_{3}^{2}\right)+\left(e_{3}-\frac{1}{3}\right)\left|u_{3}\right|^{2} \\
= & \operatorname{Re}\left\{u_{1}\left(d_{1} u_{3}+e_{1} \bar{u}_{3}\right)+d_{3} u_{3}^{2}+\left(e_{3}-\frac{1}{3}\right)\left|u_{3}\right|^{2}\right\}_{0} \leqq 0 .
\end{aligned}
$$

From (9) we derive by conjugation a linear system of equations determining $u_{1}$ in $u_{3}$ :

$$
\left\{\begin{array}{l}
u_{1}=\lambda u_{3}+\mu \bar{u}_{3} ; \\
\lambda=\frac{\bar{a}_{1} a_{1}+(1-b) \bar{e}_{1}}{(1-b)^{2}-\left|a_{1}\right|^{2}}=\frac{\bar{a}_{2}\left(a_{3}-\frac{3}{4} a_{2}^{2}\right)+2 b(1-b) a_{2}}{[2(1-b)]^{2}-\left|a_{2}\right|^{2}}, \\
\mu=\frac{\bar{a}_{1} e_{1}+(1-b) \bar{d}_{1}}{(1-b)^{2}-\left|a_{1}\right|^{2}}=\frac{b \bar{a}_{2}^{2}+2(1-b) \overline{\left(a_{3}-\frac{3}{4} a_{2}^{2}\right)}}{[2(1-b)]^{2}-\left|a_{2}\right|^{2}} .
\end{array}\right.
$$

The inequality (8) preserves its form if both sides are divided by $\left|u_{8}\right|^{2} \neq 0$. This means that we may assume without restriction

$$
\left|u_{3}\right|=1 \text {. }
$$


On this condition we express \{\}$_{0}$ in $u_{3}$ by using (11):

$$
\left\{\begin{array}{l}
\{\}_{0}=h u_{3}^{2}+k \vec{u}_{3}^{2}+l ; \\
h=d_{3}+d_{1} \lambda, \\
l=e_{1} \mu, \\
l=d_{1} \mu+e_{1} \lambda+e_{3}-\frac{1}{3} .
\end{array}\right.
$$

Thus we have

$$
\begin{aligned}
2 G= & 2 \operatorname{Re}\{\}_{0}=(h+\bar{k}) u_{3}^{2}+(\bar{h}+k) \bar{u}_{3}^{2}+2 \operatorname{Re} l \\
= & \left((h+\bar{k})^{1 / 2} u_{3}-(\bar{h}+k)^{1 / 2} \bar{u}_{3}\right)^{2}+2 \operatorname{Re} l+2|h+\bar{k}| \\
& \leqq 2 \operatorname{Re} l+2|h+\bar{k}| .
\end{aligned}
$$

Hence, the optimized bilinear $P_{3}$-inequality reads

$$
|h+\bar{k}|+\operatorname{Re} l \leqq 0
$$

and the optimizing $u_{3}$-choice is

$$
u_{3}^{2}=\left(\frac{\bar{h}+k}{h+\bar{k}}\right)^{1 / 2}=\frac{\bar{h}+k}{|\bar{h}+k|}=\frac{|h+\bar{k}|}{h+\bar{k}} .
$$

Determine now $y_{1}$ and $y_{3}$ in the optimized case. From (4) we obtain, according to (9):

$$
\begin{gathered}
y_{1}=a_{1} u_{1}+b \bar{u}_{1}+d_{1} u_{3}+e_{1} \bar{u}_{3}=\bar{u}_{1} ; \\
y_{1}=-\bar{y}_{-1} .
\end{gathered}
$$

Similarly, by the aid of (11),

$$
y_{3}=d_{1} u_{1}+\bar{e}_{1} \bar{u}_{1}+d_{3} u_{3}+e_{3} \bar{u}_{3}=\bar{u}_{3}\left[(h+\bar{k}) u_{3}^{2}+d_{1} \mu+\bar{e}_{1} \bar{\lambda}+e_{3}\right] .
$$

Here

$$
d_{1} \mu+\bar{e}_{1} \bar{\lambda}=\frac{2 \operatorname{Re}\left(a_{1} \bar{d}_{1} \bar{e}_{1}\right)+(1-b)\left(\left|e_{1}\right|^{2}+\left|d_{1}\right|^{2}\right)}{(1-b)^{2}-\left|a_{1}\right|^{2}} \in R .
$$

Because in the extremum case (13)

$$
(h+\bar{k}) u_{3}^{2}=|h+\bar{k}|=-\operatorname{Re} l,
$$

we have

$$
\begin{aligned}
& y_{3}= \bar{u}_{3}\left(-\operatorname{Re} l+d_{1} \mu+\bar{e}_{1} \bar{\lambda}+e_{3}\right) \\
&= \bar{u}_{3}\left[-\frac{1}{2}(l+\bar{l})+\frac{1}{2}\left(d_{1} \mu+\bar{e}_{1} \bar{\lambda}+\bar{d}_{1} \bar{\mu}+e_{1} \lambda\right)+e_{3}\right] \\
&= \bar{u}_{3} \operatorname{Re}\left(-l+d_{1} \mu+e_{1} \lambda+e_{3}\right)=\frac{1}{3} \bar{u}_{3} ; \\
& y_{3}=-\bar{y}_{3} .
\end{aligned}
$$


The validity of the conditions (6) in the extremum case is thus verified and (13) will hence be sharp simultaneously with the quadratic $P_{3}$-inequality. The conditions (6) written in the form

$$
y_{1}=\bar{u}_{1}, \quad y_{3}=\frac{1}{3} \bar{u}_{3}
$$

can now be directly combined with the expressions (4) to give

$$
\left\{\begin{array}{l}
a_{1} u_{1}+(b-1) \bar{u}_{1}+d_{1} u_{3}+e_{1} \bar{u}_{3}=0, \\
d_{1} u_{1}+\bar{e}_{1} \bar{u}_{1}+d_{3} u_{3}+\left(e_{3}-\frac{1}{3}\right) \bar{u}_{3}=0 .
\end{array}\right.
$$

Observe that the first condition is the same as (9), thus giving (11). Therefore, the second condition (16) assumes the form

$$
\begin{gathered}
\left(d_{1} \lambda+\bar{e}_{1} \bar{\mu}+d_{3}\right) u_{3}+\left(d_{1} \mu+\bar{e}_{1} \bar{\lambda}+e_{3}-\frac{1}{3}\right) \bar{u}_{3}=0 ; \\
d_{3}+d_{1} \lambda+\bar{e}_{1} \bar{\mu}=\left(\frac{1}{3}-e_{3}-d_{1} \mu-\bar{e}_{1} \bar{\lambda}_{2}\right) u_{3}^{-2} .
\end{gathered}
$$

Here

$$
\begin{gathered}
\frac{1}{3}-e_{3}-d_{1} \mu-\bar{e}_{1} \bar{\lambda}=\frac{1}{3}-e_{3}-\frac{1}{2}\left(d_{1} \mu+\bar{e}_{1} \bar{\lambda}+\bar{d}_{1} \bar{\mu}+e_{1} \lambda\right) \\
=\operatorname{Re}\left(\frac{1}{3}-e_{3}-d_{1} \mu-e_{1} \lambda\right)=-\operatorname{Re} l \geqq 0 .
\end{gathered}
$$

Thus, we may interprete the above $d_{3}$-condition as a circumference of the disc in which $d_{3}$ is restricted to be. - The results are collected as follows.

Result. For $S(b)$-functions with given coefficients $a_{2}$ and $a_{3}$, the number $d_{3}$ lies in the disc

$$
\left\{\begin{array}{l}
\left|d_{3}-d_{3}^{0}\right| \leqq R \\
d_{3}^{0}=-d_{1} \lambda-\bar{e}_{1} \bar{\mu}, \\
R=\frac{1}{3}-e_{3}-d_{1} \mu-\bar{e}_{1} \bar{\lambda} \geqq 0 .
\end{array}\right.
$$

The boundary points of this disc are parametrized in $u_{3}=e^{i \omega}$; thus

$$
d_{3}=d_{3}^{0}+R u_{3}^{-2} .
$$

Equality in (17) is reached in the cases where the necessary extremum condition for $F=F(z)=f\left(z^{2}\right)^{1 / 2} \in S\left(b^{1 / 2}\right)$,

$$
\begin{gathered}
\frac{1}{3} b^{3 / 2}\left(\bar{u}_{3} F^{3}-u_{3} F^{-3}\right)+b^{1 / 2}\left(\bar{s} F-s F^{-1}\right) \\
=\frac{1}{3}\left(\bar{u}_{3} z^{3}-u_{3} z^{-3}\right)+\bar{u}_{1} z-u_{1} z^{-1} ; \\
\left\{\begin{array}{l}
u_{1}=\lambda u_{3}+\mu \bar{u}_{3}, \\
s=u_{1}+\frac{1}{2} a_{2} u_{3},
\end{array}\right.
\end{gathered}
$$

defines a $S(b)$-function $f$. The abbreviations used are 
(21)

$$
\left\{\begin{array}{l}
d_{3}=\frac{1}{2} a_{4}-a_{2} a_{3}+\frac{1}{2} \frac{3}{4} a_{2}^{3}, \\
d_{1}=\frac{1}{2} a_{3}-\frac{3}{8} a_{2}^{2}, \\
e_{3}=\frac{1}{4} b\left|a_{2}\right|^{2}+\frac{1}{3} b^{3}, \\
e_{1}=\frac{1}{2} b \bar{a}_{2} ; \\
\lambda=\frac{\bar{a}_{2}\left(a_{3}-\frac{3}{4} a_{2}^{2}\right)+2 b(1-b) a_{2}}{[2(1-b)]^{2}-\left|a_{2}\right|^{2}}, \\
\mu=\frac{b \bar{a}_{2}^{2}+2(1-b) \overline{\left(a_{3}-\frac{3}{4} a_{2}^{2}\right)}}{[2(1-b)]^{2}-\left|a_{2}\right|^{2}} .
\end{array}\right.
$$

Remark. According to the above result (15) we may, in general, reduce the optimization of parameters in the Power inequality $P_{N}$ in solving the linear system of equations

$$
y_{v}=\frac{\bar{u}_{v}}{v}
$$

the number of which depends on $N$. The linear structure of this system clearly suggests that the disc result found for $N=1$ and 3 is independent of the index $N$.

\section{Rotation of the extremum function}

Consider the rotation of $f$ to $f$ :

$$
\tilde{f}(z)=\tau^{-1} f(\tau z), \quad|\tau|=1 .
$$

Suppose that $f$ is determined by a defined equation

$$
\Phi(f(z), z)=0 .
$$

Because this implies $\Phi(f(\tau z), \tau z)=0$, we see that for $\tilde{f}$

$$
\Phi(\tau \tilde{f}(z), \tau z)=0 .
$$

Apply the above observation to the condition (19), written in the form

$$
\begin{gathered}
\frac{1}{3} b^{3 / 2} \bar{u}_{3} f(z)^{3 / 2}+b^{1 / 2} \bar{s} f(z)^{1 / 2}-\left(\frac{1}{3} b^{3 / 2} u_{3} f(z)^{-3 / 2}+b^{1 / 2} s f(z)^{-1 / 2}\right) \\
=\frac{1}{3} \bar{u}_{3} z^{3 / 2}+\bar{u}_{1} z^{1 / 2}-\left(\frac{1}{3} u_{3} z^{-3 / 2}+u_{1} z^{-1 / 2}\right) .
\end{gathered}
$$

Suppose that this determines

$$
f(z)=b z+b_{2} z^{2}+\ldots
$$

which is connected with the rotated function 


$$
\begin{gathered}
\tilde{f}(z)=b z+\tilde{b}_{2} z^{2}+\ldots ; \\
\tilde{b}_{\nu}=\tau^{\nu-1} b_{\nu} .
\end{gathered}
$$

Consider now the numbers determined by (18), (20), and (21) for the rotated function $\tilde{f}$ :

$$
\begin{aligned}
& \tilde{d}_{3}=\tau^{3} d_{3}, \quad \tilde{d}_{1}=\tau^{2} d_{1}, \quad \tilde{e}_{3}=e_{3}, \\
& \tilde{e}_{1}=\tau^{-1} e_{1}, \quad \tilde{\lambda}=\tau \lambda, \quad \tilde{\mu}=\tau^{-2} \mu, \\
& \tilde{u}_{3}=\tau^{-3 / 2} u_{3}, \quad \tilde{u}_{1}=\tau^{-1 / 2} u_{1}, \quad \tilde{s}=\tilde{\tau}^{-1 / 2} s .
\end{aligned}
$$

For the function $\tilde{f}$ we thus obtain from (22)

$$
\begin{gathered}
\frac{1}{3} b^{3 / 2} \overline{\tilde{u}}_{3} \tilde{f}(z)^{3 / 2}+b^{1 / 2} \overline{\tilde{s}} \tilde{f}(z)^{1 / 2}-\left(\frac{1}{3} b^{3 / 2} \tilde{u}_{3} f(z)^{-3 / 2}+b^{1 / 2} \tilde{s} \tilde{f}(z)^{-1 / 2}\right) \\
=\frac{1}{3} \overline{\tilde{u}}_{3} z^{3 / 2}+\overline{\tilde{u}}_{1} z^{1 / 2}-\left(\frac{1}{3} \tilde{u}_{3} z^{-3 / 2}+\tilde{u}_{1} z^{-1 / 2}\right) .
\end{gathered}
$$

This means that the extremum condition is formally invariant for the rotation applied to the extremum function.

Especially, we may choose $\tilde{u}_{3}=1$, i.e.

$$
u_{3}=\tau^{3 / 2} ; \quad \tau=u_{3}^{2 / 3} .
$$

This implies for the corresponding $\tilde{F}(z)=\tilde{f}\left(z^{2}\right)^{1 / 2}$

(25) $\frac{1}{3} b^{3 / 2}\left(\tilde{F}^{3}-\tilde{F}^{-3}\right)+b^{1 / 2}\left(\overline{\tilde{s}} \tilde{F}-\tilde{s} F^{-1}\right)=\frac{1}{3}\left(z^{3}-z^{-3}\right)+\overline{\tilde{u}}_{1} z-\tilde{u}_{1} z^{-1}$.

\section{Real coefficients $a_{2}, a_{3}, a_{4}$}

In the special case where $a_{2}, a_{3}, a_{4}$ are real, the maximal $a_{4}$ and the corresponding extremum cases are studied in [1]. It is useful here to compare the real and complex cases to each other. Consider therefore the real case in detail. From (18) we deduce that if $a_{2}, a_{3}, a_{4} \in R$, then $u_{3}^{2}= \pm 1$; i.e. we may take $u_{3}=1$ or $u_{3}=i$.

The case $u_{3}=1$ was met with in [1]. The extremum condition (19) assumes the form

$$
\frac{1}{3} b^{3 / 2}\left(F^{3}-F^{-3}\right)+b^{1 / 2} s\left(F-F^{-1}\right)=\frac{1}{3}\left(z^{3}-z^{-3}\right)+u_{1}\left(z-z^{-1}\right) .
$$

Differentiation gives

$$
\begin{gathered}
b^{3 / 2} z \frac{F^{\prime}}{F}=\frac{z^{3}+z^{-3}+u_{1}\left(z+z^{-1}\right)}{F^{3}+F^{-3}+\frac{s}{b}\left(F+F^{-1}\right)} ; \\
b^{3 / 2} z \frac{F^{\prime}}{F}=\frac{F^{3}}{z^{3}} \frac{(z-i)\left(z-z_{1}\right)\left(z-z_{2}\right)(z+i)\left(z+z_{1}\right)\left(z+z_{2}\right)}{(F-i)\left(F-F_{1}\right)\left(F-F_{2}\right)(F+i)\left(F+F_{1}\right)\left(F+F_{2}\right)} ;
\end{gathered}
$$




$$
\begin{aligned}
& z_{1}, z_{2}= \pm \frac{1}{2}\left(3-u_{1}\right)^{1 / 2}+\frac{1}{2}\left(-u_{1}-1\right)^{1 / 2} \\
& F_{1}, F_{2}= \pm \frac{1}{2}\left(3-\frac{s}{b}\right)^{1 / 2}+\frac{1}{2}\left(-\frac{s}{b}-1\right)^{1 / 2} .
\end{aligned}
$$

Denote $z_{v}=e^{i \varphi}, F_{v}=e^{i \varphi}(v=1$ or 2$)$. Because $z_{\nu}^{2}+z_{v}^{-2}-1+u_{1}=$ $0, F_{\nu}^{2}+F_{\nu}^{-2}-1+s / b=0$, we have

$$
u_{1}=1-2 \cos 2 \varphi_{v}, \quad \frac{s}{b}=1-2 \cos 2 \psi_{v} .
$$

This implies

$$
\begin{aligned}
& -1 \leqq u_{1} \leqq 3 \\
& -1 \leqq \frac{s}{b} \leqq 3 .
\end{aligned}
$$

(28) is necessary and sufficient for four unit roots $z_{\nu}$. Similarly, (29) is connected with four unit roots $F_{y}$.

If (29) holds, then $-1 \leqq s / b$ implies

$$
-b-\frac{1}{2} a_{2} \leqq u_{1} \text {. }
$$

Because $-(1-b) \leqq-a_{2} / 2$, it follows from the preceding condition that

$$
-1=-b-(1-b) \leqq u_{1}
$$

i.e. the left side of (29) implies the left side of (28). - The factorized condition (27) suggests the type $1^{\circ}$ in Figure 1 for the extremum domain.

If $s / b>3$, the type $2^{\circ}$ of Figure 1 emerges from the factorized condition.

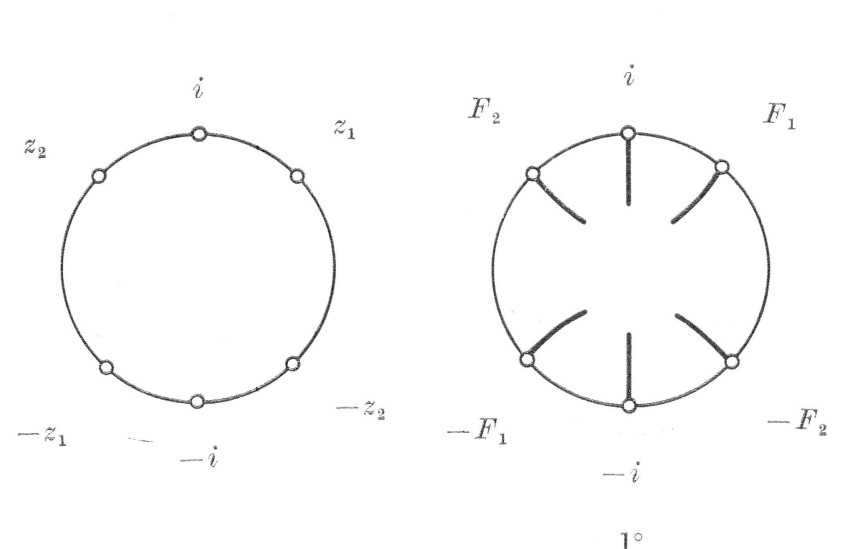

$1^{\circ}$

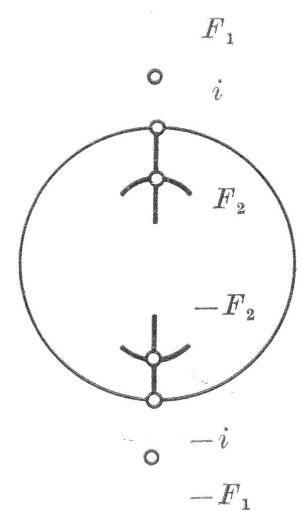

$2^{\circ}$

Figure 1. 
The type of the possible extremum domain can be further studied by the aid of boundary correspondence defined by (26). Take $z=e^{i \varphi}$ and denote

$$
F\left(e^{i \varphi}\right)=r(\varphi) e^{i \psi(\varphi)}
$$

in (26), which gives

$$
\left\{\begin{array}{l}
\left(r-r^{-1}\right)\left[\frac{1}{3} b\left(r^{2}+1+r^{-2}\right) \cos 3 \psi+s \cos \psi\right]=0 \\
A=\frac{1}{2}\left[\frac{1}{3} b^{3 / 2}\left(r^{3}+r^{-3}\right) \sin 3 \psi+b^{1 / 2} s\left(r+r^{-1}\right) \sin \psi\right] \\
\quad=\frac{1}{3} \sin 3 \varphi+u_{1} \sin \varphi=B .
\end{array}\right.
$$

For $F\left(e^{i \varphi}\right)$ the first condition (30) implies either that $r(\psi) \equiv 1$ or that $r(\psi)$ satisfies the condition

$$
\left\{\begin{array}{l}
H=\frac{1}{3} b\left(r^{2}+1+r^{-2}\right)=-\frac{1}{1-4 \sin ^{2} \psi}=K, \text { or } \\
\cos \psi \equiv 0 .
\end{array}\right.
$$

The boundary curve candidates are given schematically in Figure 2.

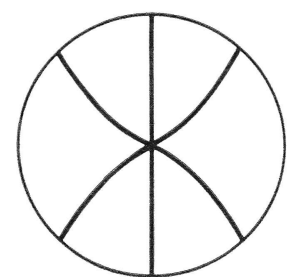

$0 \leqq \frac{1}{3} s<b$

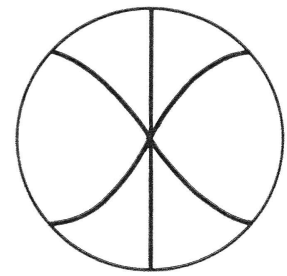

$0 \leqq-s<b$

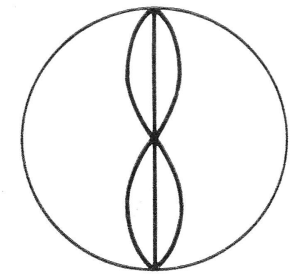

$\frac{1}{3} s=b$

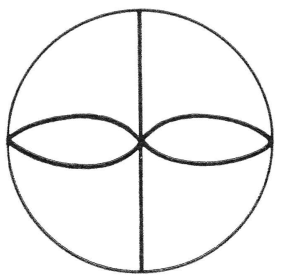

$\cdots s=b$

Figure 2.

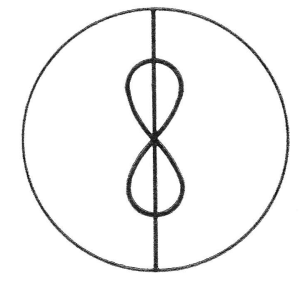

$\frac{1}{3} s>b$

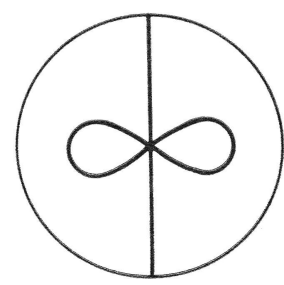

$-s>b$ 
It appears that the first condition (30) is connected with the type $1^{\circ}$ if $-s / b \leqq 1$ and $s / b \leqq 3$, i.e. if (29) holds. The type $2^{\circ}$ occurs for $s / b>3$.

Up to now we have found the following conditions necessary for the existence of the boundary mapping

$$
-1 \leqq \frac{s}{b} \text { and } u_{1} \leqq 3
$$

If $a_{2}, a_{3} \in R$ and $u_{1} \leqq 3$ we have

$$
u_{1}=\lambda+\mu=\frac{a_{3}-\frac{3}{4} a_{2}^{2}+b a_{2}}{2(1-b)-a_{2}} \leqq 3,
$$

which gives us the domain bounded by the parabola $u_{1}=3$.

The type $1^{\circ}$ is connected with values for which $-1 \leqq s / b \leqq 3$, i.e.

$$
-b \leqq \frac{a_{3}-\frac{5}{4} a_{2}^{2}+a_{2}}{2(1-b)-a_{2}} \leqq 3 b .
$$

This defines the domain bounded by two parabolas $s=-b$ and $s=3 b$.

- All the limiting parabolas mentioned meet at the point

$$
a_{2}=2(1-b), \quad a_{3}=3-8 b+5 b^{2}
$$

connected with the radial slit mapping.

The type $2^{\circ}$ occurs if $s>3 b$, i.e. in the domain bounded by the parabolas $s=3 b$ and $u_{1}=3$. These domains are illustrated in Figure 7 of [1], where the notation $u$ means the same as $u_{1}$ here.

There remains the second condition (30) which gives for $r(\psi) \equiv 1$

$$
A=\frac{1}{3} b^{3 / 2} \sin 3 \psi+b^{1 / 2} s \sin \psi=\frac{1}{3} \sin 3 \varphi+u_{1} \sin \varphi=B .
$$

For the boundary correspondence to be of the type $1^{\circ}$ or $2^{\circ}$ it is sufficient that each $\psi \in[0,2 \pi]$ has a uniquely determined pre-image $\varphi \in[0,2 \pi]$. The correspondence becomes clear from Figures 3 and 4.

Consider first the alternative $1^{\circ}$, connected with Figure 3 . There are two conditions to be satisfied for the existence of a pre-image of each $\psi$.

1)

$$
\begin{gathered}
\frac{1}{3}\left(u_{1}+1\right)^{3 / 2} \geqq \frac{1}{3}(s+b)^{3 / 2} \\
1-b \leqq s-u_{1}=\frac{1}{2} a_{2} .
\end{gathered}
$$

This condition is always true 
166

RONALD KORTRAM and OLII TAMMI
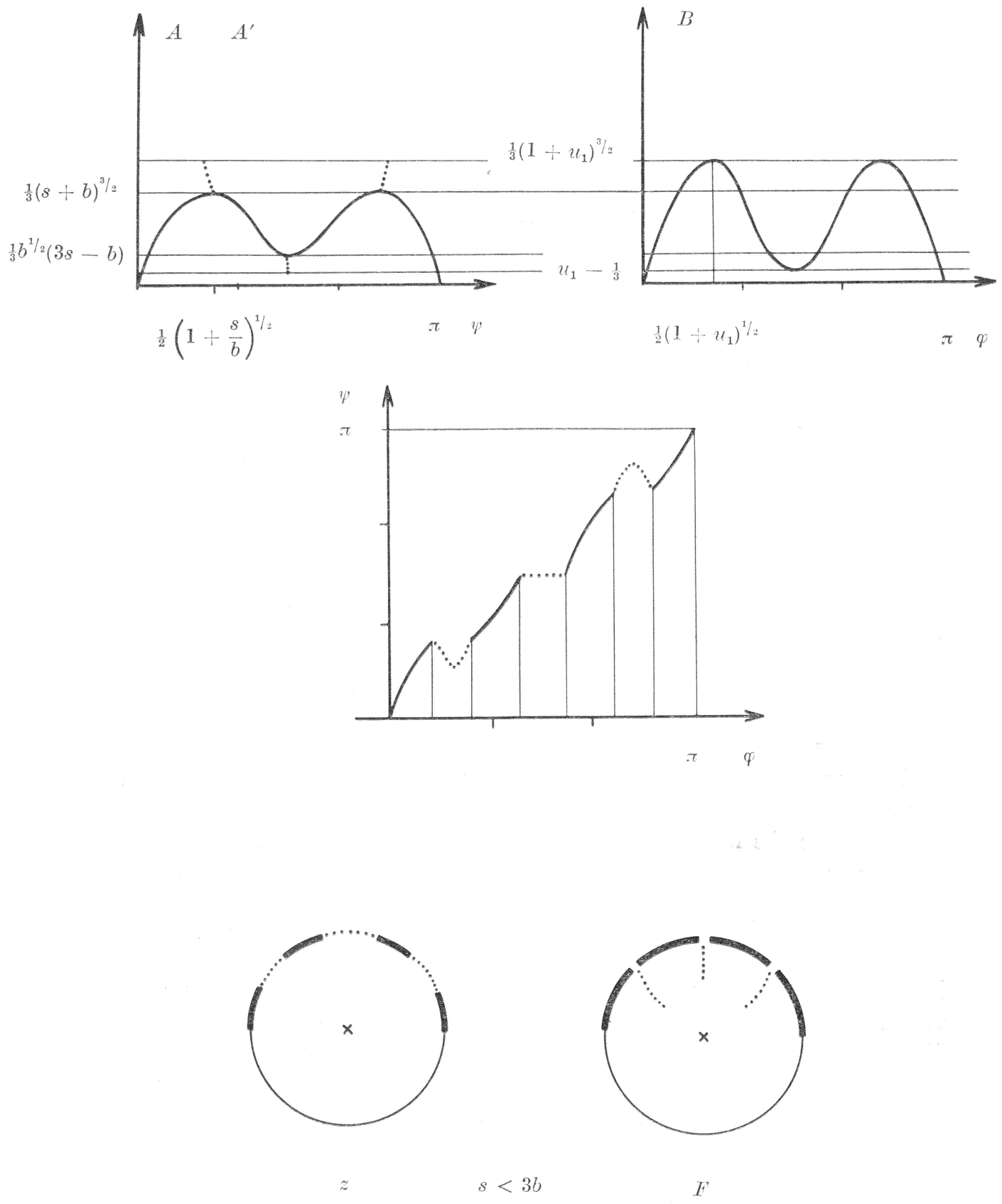

Figure 3. 

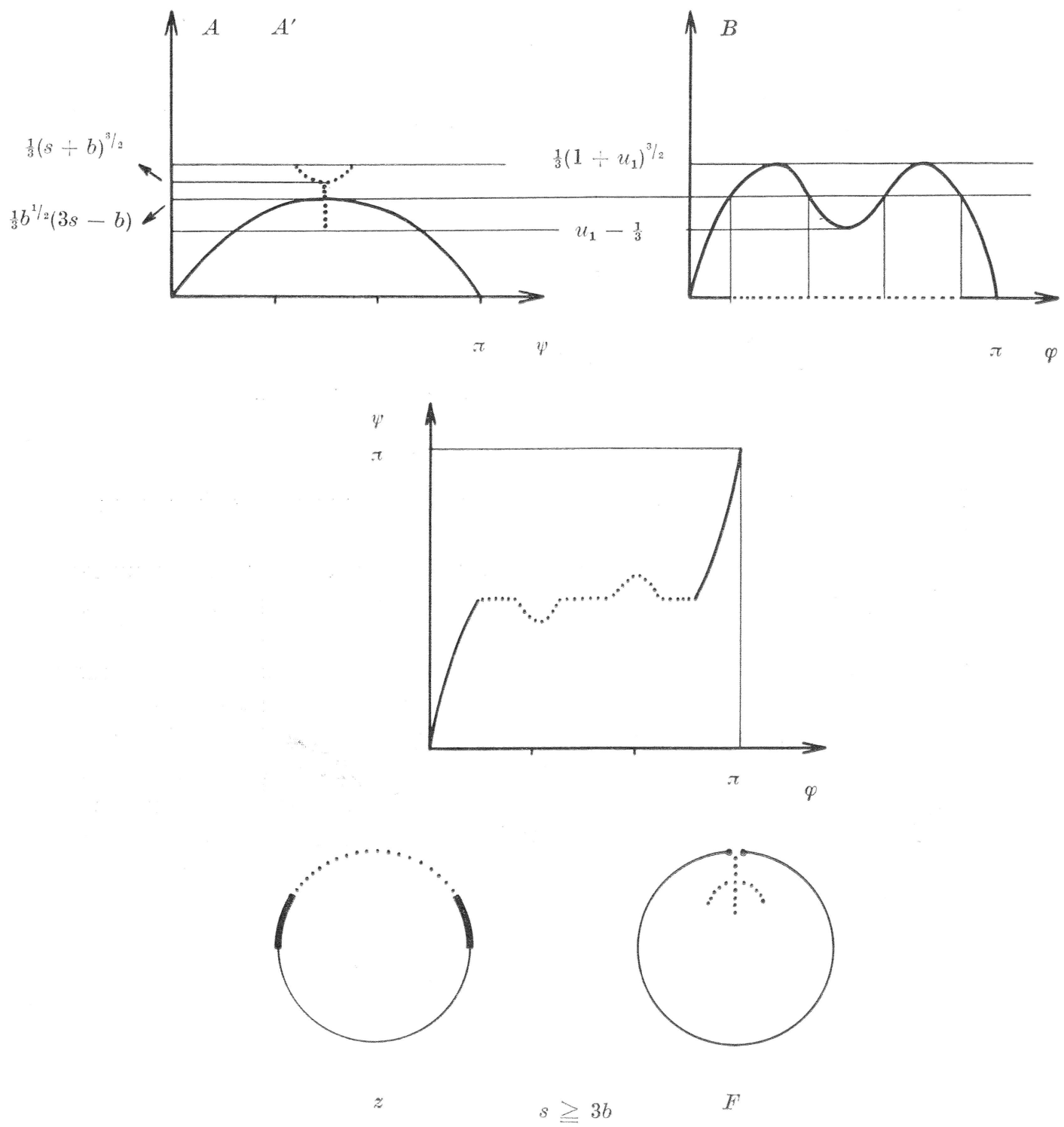

Figure 4.

2)

$$
\begin{gathered}
u_{1}-\frac{1}{3} \leqq \frac{1}{3} b^{1 / 2}(3 s-b) \\
u_{1} \leqq \frac{1}{3}\left(1+b^{1 / 2}+b\right)+\frac{1}{2} \frac{b^{1 / 2}}{1-b^{1 / 2}} a_{2} .
\end{gathered}
$$


Because $\left|a_{2}\right| \leqq 2(1-b)$, this implies $u_{1} \leqq 3$. According to (33), the condition can be rewritten in the form

$$
a_{3} \leqq \frac{1}{4} \frac{3-5 b^{1 / 2}}{1-b^{1 / 2}} a_{2}^{2}-\frac{1}{3}\left(1-b^{1 / 2}\right)^{2} a_{2}+\frac{2}{3}\left(1+b^{1 / 2}\right)\left(1-b^{3 / 2}\right) .
$$

The points of the slits where $\cos \psi \neq 0$ are controlled by the last equation (30) and (31), which give

$$
\left\{\begin{aligned}
\varkappa & =r+r^{-1}=(1-v)^{1 / 2} \\
v & =\frac{3 s}{b} \frac{1}{1-4 \sin ^{2} \psi}, \\
A^{\prime} & =\frac{1}{2}\left[\frac{1}{3} b^{3 / 2}\left(\varkappa^{3}-3 \varkappa\right) \sin 3 \psi+b^{1 / 2} s \varkappa \sin \psi\right] \\
& =\frac{1}{3} \sin 3 \varphi+u_{1} \sin \varphi=B .
\end{aligned}\right.
$$

The dotted arcs in Figure 3 indicate points connected with the slits, determined by (39).

As a result of the above considerations we have found a triangle bounded by the parabolas $s=-b, s=3 b$ and (38), in the coefficient body $\left(a_{3}, a_{2}\right)$ where the extremum condition (26) defines functions $F \in S\left(b^{1 / 2}\right)$ of the type $1^{\circ}$. The corresponding functions $f \in S(b)$ are thus 3-slit functions. On the boundary curves of the triangle there exist certain degenerated limit cases of those 3 -slit functions. The type of these is readable in Figure 3.

The alternative $2^{\circ}$ is connected with Figure 4 . In this case $A(\psi)$ has only one maximum in the interval $[0, \pi]$ which is bigger than minimum of $B(\varphi)$, provided that (37) continues to hold. This order is needed to guarantee the 3 -fork structure of the slit. Again, the dotted arcs belong to the slits and are governed by $A^{\prime}(\psi)$ of (39). Especially the branch point is connected with

$$
\left\{\begin{array}{l}
r=\frac{(s+b)^{1 / 2}-(s-3 b)^{1 / 2}}{2 b^{1 / 2}}, \\
\psi=\frac{\pi}{2},
\end{array}\right.
$$

obtained from $F_{2}$ of $(27)$. Thus $\varkappa=(s / b+1)^{1 / 2}$ and (39) give

$$
A^{\prime}\left(\frac{\pi}{2}\right)=\frac{1}{3}(b+s)^{3 / 2} .
$$

This is $\leqq \frac{1}{3}\left(u_{1}+1\right)^{3 / 2}$, which condition, according to (36), is always true.

Thus, in the case $2^{\circ}$ the parabola $s=3 b$ together with the parabola (38) determines a domain in the coefficient body $\left(a_{3}, a_{2}\right)$, where the functions $f \in S(b)$ given by (26) are 3 -fork-slit functions, Actually, this type 
continues to hold slightly behind the parabola (38). The bounadry curve on which the extremum function degenerates into a 2 -fork-slit function is given by

$$
-\frac{1}{3}+u_{1} \leqq \frac{1}{3}(b+s)^{3 / 2} .
$$

This defines a third degree arc in $a_{2}, a_{3}$ with the end points at the intersection of the curves

$$
\left\{\begin{array}{l}
s=3 b, \\
-\frac{1}{3}+u_{1}=\frac{1}{3} b^{1 / 2}(3 s-b) .
\end{array}\right.
$$

Thus, these end points are

$$
\begin{aligned}
& \left\{\begin{array}{l}
a_{2}=-\frac{2}{3}\left(1-9 b+8 b^{3 / 2}\right), \\
a_{3}=\frac{5}{9}\left(1-9 b+8 b^{3 / 2}\right)^{2}+(3 b+1) \frac{2}{3}\left(1-9 b+8 b^{3 / 2}\right)+6\left(b-b^{2}\right) ;
\end{array}\right. \\
& \left\{\begin{array}{l}
a_{2}=2(1-b), \\
a_{3}=3-8 b+5 b^{2} .
\end{array}\right.
\end{aligned}
$$

In the case $b=1 / 2$ the curve $(38)^{\prime}$ differs very little from that defined by (38). Hence, the Figure 7 of [1] continues to give a schematic presentation of the extremum domain.*

In the extremum domain, defined above by (32), (38) and $(38)^{\prime}$ the inequality (17) is sharp and we obtain from (18) the maximum for $d_{3}$ :

$$
\begin{aligned}
\max d_{3} & =d_{3}^{0}+R=-d_{1} \lambda-e_{1} \mu+1 / 3-e_{3}-d_{1} \mu-e_{2} \lambda \\
& =-\left(d_{1}+e_{1}\right)(\lambda+\mu)+1 / 3-e_{3} \\
& =\frac{1}{3}\left(1-b^{3}\right)-\frac{1}{4} b a_{2}^{2}-\frac{1}{2} \frac{\left(a_{3}-\frac{3}{4} a_{2}^{2}+b a_{2}\right)^{2}}{2(1-b)-a_{2}} \\
& =M\left(a_{2}, a_{3}\right) .
\end{aligned}
$$

This is the result derived already in [1]. From the above inequality $\min d_{3}$ also follows. Instead of $f(z)$ consider

$$
-f(-z)=b z-b_{2} z^{2}+b_{3} z^{3}-b_{4} z^{4}+\ldots .
$$

This means the following transformation:

$$
f(z), a_{2}, a_{3}, a_{4}, d_{3} \Rightarrow-f(-z),-a_{2}, a_{3},-a_{4},-d_{3} .
$$

Thus,

$$
-d_{3}\left(a_{2}, a_{3}\right) \leqq M\left(-a_{2}, a_{3}\right)
$$

i.e.

* In this context another inaccuracy of [1] can be mentioned. Unfortunately the direction of rotation of the extremum domains in Figure 12 of [1] is reversed. 


$$
-M\left(-a_{2}, a_{3}\right) \leqq d_{3}\left(a_{2}, a_{3}\right) .
$$

Here the point $\left(-a_{2}, a_{3}\right)$ must lie in the domain defined above. Thus the range of $\left(a_{2}, a_{3}\right)$ for which (41) is sharp is symmetric with the preceding domain with respect to the $a_{3}$ axis.

We may check that our general result (17) implies (41) by substituting $u_{3}=i$ in (18) for $a_{2}, a_{3}, a_{4} \in R$ :

$$
\begin{aligned}
d_{3} & \geqq d_{3}^{0}-R=-d_{1} \lambda-e_{1} \mu-1 / 3+e_{3}+d_{1} \mu+e_{1} \lambda \\
& =\left(d_{1}-e_{1}\right)(\mu-\lambda)-1 / 3+e_{3} \\
& =-\frac{1}{3}\left(1-b^{3}\right)+\frac{1}{4} b a_{2}^{2}+\frac{1}{2} \frac{\left(a_{3}-\frac{3}{4} a_{2}^{2}-b a_{2}\right)^{2}}{2(1-b)+a_{2}} \\
& =-M\left(-a_{2}, a_{3}\right) .
\end{aligned}
$$

\section{The special case $a_{2}=0$}

Before discussing the general case by the aid of numerical examples, we want to find an easy case, different from the preceding one, where boundary points of the $d_{3}$-disc give a sharp inequality. For $a_{2}=0$, $a_{3} \neq 0 \quad(21)$ gives

$$
\left\{\begin{array}{l}
d_{3}=\frac{1}{2} a_{4}, \quad d_{1}=\frac{1}{2} a_{3}, \quad e_{3}=\frac{1}{3} b^{3}, \quad e_{1}=0 ; \\
\lambda=0, \quad \mu=\frac{\bar{a}_{3}}{2(1-b)}, \quad u_{1}=\mu \bar{u}_{3}=\frac{\bar{a}_{3}}{2(1-b)} u_{3}^{-1} .
\end{array}\right.
$$

Now $F$ is determined by (19). Apply to it the rotation $\tau=u_{3}^{2 / 3}$, giving $\tilde{u}_{3}=1$. The rotated function $\tilde{f}$ has coefficients $\tilde{a}_{v}$ and defines $\tilde{F}=\tilde{f}\left(z^{2}\right)^{1 / 2}$, for which there holds, according to (25),

$$
\begin{aligned}
& \frac{1}{3} b^{3 / 2}\left(\tilde{F}^{3}-\tilde{F}^{-3}\right)+\frac{b^{1 / 2}}{2(1-b)}\left(\overline{\tilde{a}}_{3} \tilde{F}-\tilde{a}_{3} \tilde{F}^{-1}\right) \\
& =\frac{1}{3}\left(z^{3}-z^{-3}\right)+\frac{1}{2(1-b)}\left(\overline{\tilde{a}}_{3} z-\tilde{a}_{3} z^{-1}\right) .
\end{aligned}
$$

Now choose $\tau$ such that

$$
\tilde{a}_{3}=\tau^{2} a_{3}=\left|a_{3}\right|,
$$

i.e.

$$
\tau=\left(\frac{\left|a_{3}\right|}{a_{3}}\right)^{1 / 2}, \quad u_{3}^{2}=\tau^{3}=\left(\frac{\left|a_{3}\right|}{a_{3}}\right)^{3 / 2} .
$$


At the boundary point we obtain from (18) for $d_{3}$

$$
d_{3}=\frac{1}{2} a_{4}=\left[\frac{1}{3}\left(1-b^{3}\right)-\frac{\left|a_{3}\right|^{2}}{4(1-b)}\right]\left(\frac{a_{3}}{\left|a_{3}\right|}\right)^{3 / 2},
$$

and $\tilde{F}$ satisfies the condition

$$
\left\{\begin{array}{l}
\frac{1}{3} b^{3 / 2}\left(\tilde{F}^{3}-\tilde{F}^{-3}\right)+b^{1 / 2} s\left(\tilde{F^{1}}-\tilde{F}^{-1}\right)=\frac{1}{3}\left(z^{3}-z^{-3}\right)+s\left(z-z^{-1}\right), \\
s=\frac{\left|a_{3}\right|}{2(1-b)} .
\end{array}\right.
$$

Thus, we may use the results true for the equation (26). Both the first condition (32) and (38) must be satisfied. In the present case $-1 \leqq s / b$ is automatically true and (38) reduces into the form

$$
\left|a_{3}\right| \leqq \frac{2}{3}\left(1+b^{1 / 2}\right)\left(1-b^{3 / 2}\right) .
$$

Hence we see that if $a_{2}=0$ and $a_{3}$ satisfies (44), the corresponding boundary point of the $d_{3}$-disc is sharp in the sense that (43) holds at this boundary point.

\section{Real coefficients $a_{2}, a_{3}$}

In the general case, where all the coefficients $a_{2}, a_{3}, a_{4}$ are complex, we may normalize the mapping by rotation into the form (25). For brevity, drop tilda in the notations. Differentiation gives

$$
b^{3 / 2} z \frac{F^{\prime}}{F^{\prime}}=\frac{z^{3}+z^{-3}+\bar{u}_{1} z+u_{1} z^{-1}}{F^{3}+F^{-3}+b^{-1} \bar{s} F^{-1} s F^{-1}} .
$$

The factorization depends on the roots of the equation having the type

$$
z^{3}+z^{-3}+\bar{c} z+c z^{-1}=0 \text {. }
$$

If $z$ is a root here, so are $-z, \bar{z}^{-1}$ and $-\bar{z}^{-1}$. This includes two alternatives.

$1^{\circ}$. If there are two unit roots (i.e. they have the absolute value 1) not lying on the same diameter of the unit circle $|z|=1$, there must be four, and hence six, unit roots.

$2^{\circ}$. If $z$ is a non-unit root $(|z| \neq 1)$, there are four non-unit roots on the diameter where $z$ lies and two unit roots again on one diameter. This implies that the type of domains obtained is the same as before, except that they are not twice axially symmetric any more.

We are not too far from the most general case if we assume $a_{2}$ and $a_{3}$ to be real and $a_{4}$ complex. In Section 4 we actually studied those 
boundary points of the $d_{3}$-disc which were connected with that special case and were given by the values $u_{3}=1$ and $u_{3}=i$. Therefore we may restrict ourselves to the values

$$
u_{3}=e^{i \omega}, \quad \omega \in\left(0, \frac{\pi}{2}\right) .
$$

For brevity, normalize by the rotation where

$$
\tau=u_{3}^{2 / 3}=e^{i \frac{2 \omega}{3}} .
$$

The connection (25) holds for $\tilde{F}$. The coefficients $\tilde{u}_{1}$ and $\tilde{s}$ are determined by the following formulae:

$$
\begin{aligned}
& \left\{\begin{array}{l}
u_{1}=\lambda e^{i \omega}+\mu e^{-i \omega}=(\lambda+\mu) \cos \omega+i(\lambda-\mu) \sin \omega, \\
s=u_{1}+\frac{1}{2} a_{2} e^{i \omega}=\left(\lambda+\mu+\frac{1}{2} a_{2}\right) \cos \omega+i\left(\lambda-\mu+\frac{1}{2} a_{2}\right) \sin \omega ;
\end{array}\right. \\
& \left\{\begin{array}{l}
\tilde{u}_{1}=u_{3}^{-1 / 3} u_{1}=\xi e^{i \eta} \\
\tilde{s}=u_{3}^{-1 / 3} s=\sigma e^{i \chi}
\end{array}\right. \\
& \left\{\begin{array}{l}
\lambda+\mu=\frac{a_{3}-\frac{3}{4} a_{2}^{2}+b a_{2}}{2(1-b)-a_{2}} \\
\lambda-\mu=\frac{a_{3}-\frac{3}{4} a_{2}^{2}-b a_{2}}{2(1-b)+a_{2}} \\
\lambda+\mu+\frac{1}{2} a_{2}=\frac{a_{3}-\frac{5}{4} a_{2}^{2}+a_{2}}{2(1-b)-a_{2}}, \\
\lambda-\mu+\frac{1}{2} a_{2}=\frac{a_{3}-\frac{1}{4} a_{2}^{2}+(1-2 b) a_{2}}{2(1-b)+a_{2}} .
\end{array}\right.
\end{aligned}
$$

The numbers $\xi, \sigma ; \eta, \chi$ are determined as follows:

$$
\begin{aligned}
& \left\{\begin{array}{l}
\xi^{2}=(\lambda+\mu)^{2} \cos ^{2} \omega+(\lambda-\mu)^{2} \sin ^{2} \omega, \\
\sigma^{2}=\left(\lambda+\mu+\frac{1}{2} a_{2}\right)^{2} \cos ^{2} \omega+\left(\lambda-\mu+\frac{1}{2} a_{2}\right)^{2} \sin ^{2} \omega
\end{array}\right. \\
& \eta=\arg u_{1}-\omega / 3 ;\left\{\begin{array}{l}
\cos \arg u_{1}=\frac{\lambda+\mu}{\xi} \cos \omega, \\
\sin \arg u_{1}=\frac{\lambda-\mu}{\xi} \sin \omega .
\end{array}\right. \\
& \chi=\arg s-\omega / 3 ;\left\{\begin{array}{l}
\cos \arg s=\frac{\lambda-\mu+\frac{1}{2} a_{2}}{\sigma} \cos \omega, \\
\sin \arg s=\frac{\lambda-\mu+\frac{1}{2} a_{2}}{\sigma} \sin \omega .
\end{array}\right.
\end{aligned}
$$


By substituting in (25) $z=e^{i \varphi}, \tilde{F}=r e^{i \varphi}$, and splitting it into real and imaginary parts, we end up with the conditions

$$
\left\{\begin{array}{l}
\frac{1}{3} b^{3 / 2}\left(r^{3}-r^{-3}\right) \cos 3 \psi+b^{1 / 2} \sigma\left(r-r^{-1}\right) \cos (\psi-\chi)=0 \\
\frac{1}{3} b^{3 / 2}\left(r^{3}+r^{-3}\right) \sin 3 \psi+b^{1 / 2} \sigma\left(r+r^{-1}\right) \sin (\psi-\chi) \\
=\frac{2}{3} \sin 3 \varphi+2 \xi \sin (\varphi-\eta) .
\end{array}\right.
$$

The first condition gives a unit circumference $r=1$ and, for $r<1$, curves in the disc $|\tilde{F}|<1$, determined by the equation

$$
H=\frac{1}{3} b\left(r^{2}+1+r^{-2}\right)=-\sigma \frac{\cos (\psi-\chi)}{\cos 3 \psi}=K .
$$

The second condition (48) defines for $r=1$ the connection between the unit circumferences in the $z$ - and $\tilde{F}$-planes. Especially, for $r=1$ each $\psi \in[0,2 \pi]$ must obtain a uniquely determined pre-image $\varphi \in[0,2 \pi]$, according to the connection

$$
\begin{aligned}
A & =\frac{1}{3} b^{3 / 2} \sin 3 \psi+b^{1 / 2} \sigma \sin (\psi-\eta) \\
& =\frac{1}{3} \sin 3 \varphi+\xi \sin (\varphi-\eta)=B .
\end{aligned}
$$

For $r<1,(49)$ together with the second condition (48) determines, the connection between $\varphi$ and $\psi$ on the slits. From (49) we deduce analogously to (39) in Section 4, that

$$
\left\{\begin{array}{l}
x=r+r^{-1}=(1-v)^{1 / 2} \\
v=\frac{3 \sigma \cos (\psi-\chi)}{b} \frac{\cos 3 \psi}{}
\end{array}\right.
$$

The second condition (48) thus assumes the form

$$
\begin{aligned}
A^{\prime} & =\frac{1}{2}\left[\frac{1}{3} b^{3 / 2}\left(x^{3}-3 \varkappa\right) \sin 3 \psi+b^{1 / 2} \sigma x \sin (\psi-\chi)\right] \\
& =\frac{1}{3} \sin 3 \varphi+\xi \sin (\varphi-\eta)=B .
\end{aligned}
$$

For each fixed $\omega$ and the given pair $\left(a_{2}, a_{3}\right)$ we can study the above conditions similarly to the previous case $\omega=0$ (and $\omega=\pi / 2$ ). However, the solutions of the extremum problems involved are, in general, only numerically possible.

In the case

$$
b=\frac{1}{2}, \quad a_{2}=a_{3}=\frac{1}{4}, \quad \omega=\pi / 4
$$

the above connections are illustrated in Figure 5 by the aid of graphs determined numerically. In Figure 6 there is the resulting extremum domain. 


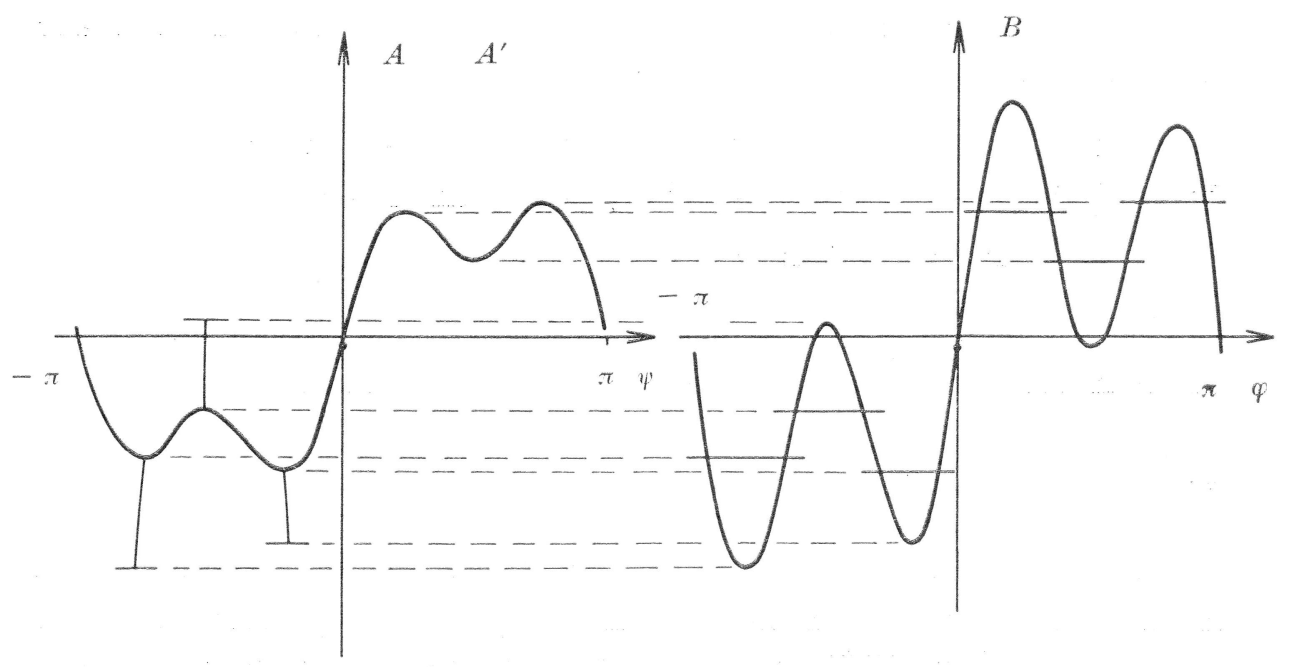

Figure 5.

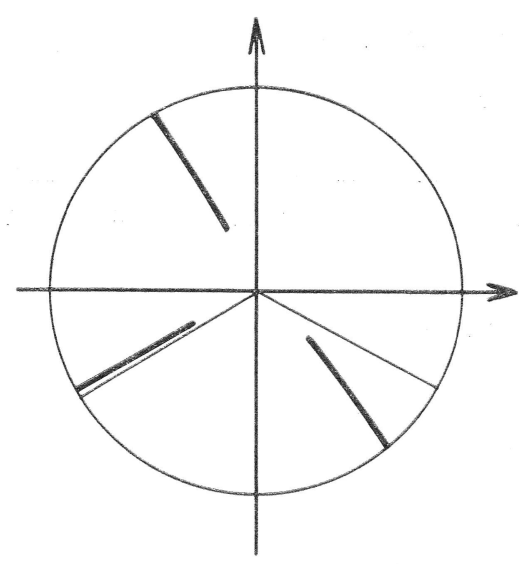

$f$

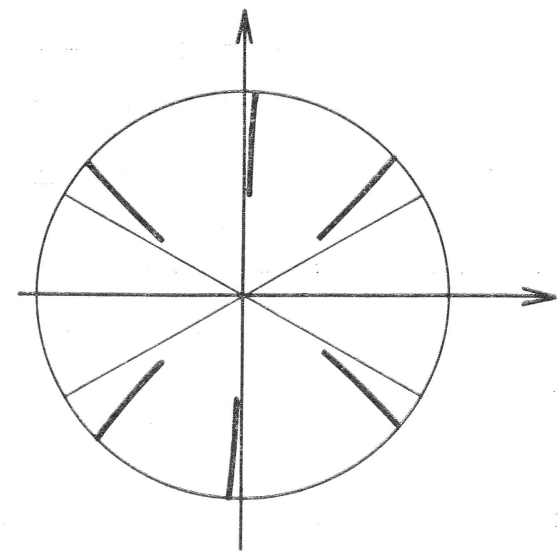

$\widetilde{F}$

Figure 6 .

By determining the range of $\left(a_{2}, a_{3}\right)$ for various values of $\omega$, one could obtain, as an intersection, the domain round the origin where all the boundary points of the $d_{3}$-dise are reached.

\section{References}

[1] KorTram, R., and O. TAMMI: On the first coefficient regions for bounded univalent functions. - Ann. Acad. Sci. Fenn. Ser. A I 592, 1974, 1-26. 
[2] SChIFFER, M., and O. TAMmI: A Green's inequality for the power matrix. Ibid. 501, 1971, 1-15.

[3] Tamm, O.: On optimizing parameters of the Power inequality for $a_{4}$ in the class of bounded univalent functions. - Ibid. 560, 1973, 1-24.

University of Nijmegen

Department of Mathematics

Nijmegen

The Netherlands

Received 2 December 1974
University of Helsinki

Department of Mathematics

SF-00100 Helsinki 10

Finland 